\title{
Method for polarization shaping at free-electron lasers
}

\author{
Svitozar Serkez $\odot,{ }^{1, *}$ Andrei Trebushinin $\odot,{ }^{2,3}$ Mykola Veremchuk $\odot,{ }^{4}$ and Gianluca Geloni ${ }^{1}$ \\ ${ }^{1}$ European XFEL, 4 Holzkoppel street, 22869 Schenefeld, Germany \\ ${ }^{2}$ Budker Institute of Nuclear Physics, 11 Lavrentieva avenue, 630090 Novosibirsk, Russia \\ ${ }^{3}$ Novosibirsk State University, 2 Pirogova street, 630090 Novosibirsk, Russia \\ ${ }^{4}$ Physics Department, Taras Shevchenko Kyiv University, 2 Academician Glushkov avenue, \\ Kyiv 03022, Ukraine
}

(Received 29 July 2019; published 27 November 2019)

\begin{abstract}
$\mathrm{X}$-ray free electron lasers provide short, polarized, high-power pulses of $\mathrm{x}$-ray radiation, where polarization properties are determined by the undulator magnetic field. We propose a cost-effective method to shape the polarization of FEL radiation on the sample both spatially, on a few- $\mu \mathrm{m}$ scale, and/or temporally on the 100-femtosecond scale. The method is based on coaxial superposition of two coherent radiation pulses with different frequencies emitted in two consequent undulators set to emit radiation with orthogonal polarization states. Its capabilities are demonstrated via numerical simulations for the SASE3 undulator line of the European X-ray Free-Electron Laser. Generation of x-ray free-electron laser pulses with shaped polarization may be interesting for studies of magnetism or ultrafast phenomena and will facilitate further developments of complex light engineering in the soft X-ray range.
\end{abstract}

DOI: 10.1103/PhysRevAccelBeams.22.110705

\section{INTRODUCTION}

Free electron lasers (FELs) opened up the possibility of obtaining polarized $\mathrm{x}$-ray pulses with unprecedented power and femtosecond-order duration [1].

Generation of structured light and, in particular, radiation with structured polarization, has interesting prospects for the future, as outlined in [2] and references therein. Techniques for generating radiation pulses with complex polarization topology are well developed in the optical range; for instance, generation of radiation with spatially varying ellipticity [3], full Poincaré beams [4], polarization Möbius strips [5], and radiation with temporally shaped polarization [6] were reported. Passing optical light through numerous components is necessary to achieve such results.

However, it is challenging to apply transmissive optical elements in the soft x-ray range due to strong absorption. In this paper we propose a simple method to shape the polarization state of the FEL radiation using only components already developed for such facilities, namely helical undulators, phase-shifters, longitudinal wakefield structures [7], and reflective x-ray mirrors.

\footnotetext{
*svitozar.serkez@xfel.eu
}

Published by the American Physical Society under the terms of the Creative Commons Attribution 4.0 International license. Further distribution of this work must maintain attribution to the author(s) and the published article's title, journal citation, and DOI.
When an electron beam with coherently modulated density passes through the undulator resonant to the modulation frequency, a coherent radiation pulse is emitted. One can call such device a radiator. Polarization properties of that pulse are determined by the trajectory of electrons in the radiator, which in turn depends on the undulator magnetic field. As all electrons within the electron beam travel with nearly the same trajectory, the generated radiation pulses have homogeneous polarization properties: within the resonance approximation planar undulators yield linearly polarized radiation, while circularly polarized radiation can be generated in helical undulators $[8,9]$.

When an electron beam passes consequently through two radiators set to emit radiation with orthogonal polarization states, the resulting on-axis polarization state will be located somewhere on the large circle of the Poincaré sphere between the original polarization states ${ }^{1}$ as illustrated on Fig. 1. Its location along the circle is determined by the phase difference $\Delta \phi$ between the beams.

This scheme was first proposed in [10] as a way of generating circularly polarized radiation at synchrotron facilities $^{2}$, as illustrated on Fig. 2(a). Later this approach was extended for FELs and circular polarization basis [11], as illustrated on Fig. 2(b), experimentally demonstrated for crossed planar and helical undulators [12-15]

\footnotetext{
${ }^{1}$ Here we assume that the pulses are overlapped both spatially and temporally.

${ }^{2}$ The monochromator was used in order to provide the necessary longitudinal coherence.
} 


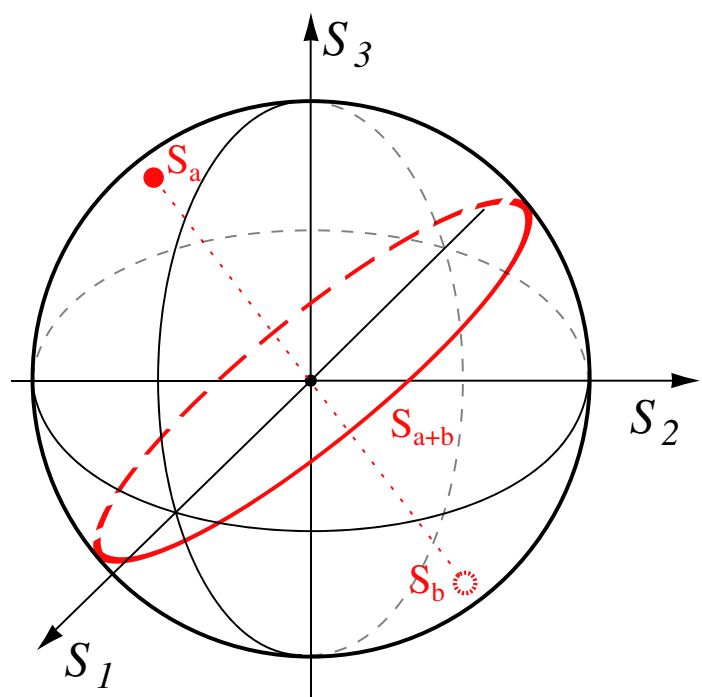

FIG. 1. Combination of radiation pulses with orthogonal polarization basis $S_{a}$ and $S_{b}$, represented on Poincaré sphere, yields polarization states located on a big circle of sphere.

and is currently referred to as the "crossed undulator technique."

In order for this method to work, it is important that the phase difference $\Delta \phi$ between the two polarized pulses is kept fixed both transversely and in time. Since both pulses are emitted by the same electron beam, this scenario would be naturally satisfied if not for the slippage of the radiation with respect to the electron beam in the undulator. If comparable or larger than the radiation coherence time, this slippage does deteriorate polarization properties [16]. Therefore it is challenging to apply this technique for self-amplified spontaneous emission (SASE) operation mode when radiation coherence time, typically much smaller than the pulse duration, is comparable to the radiation slippage.

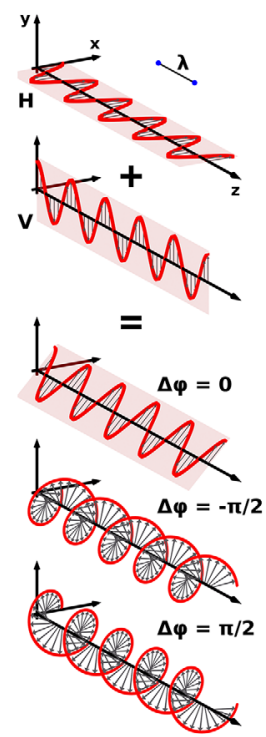

(a) sum of linearly polarized beams
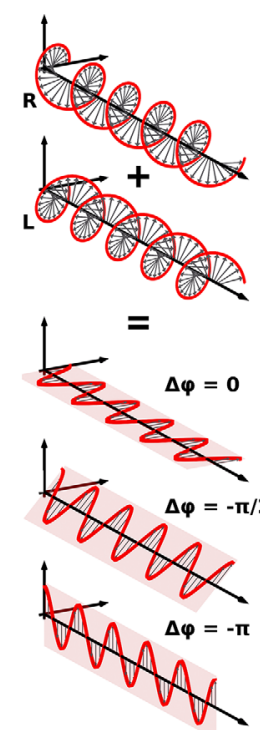

(b) sum of circularly polarized beams
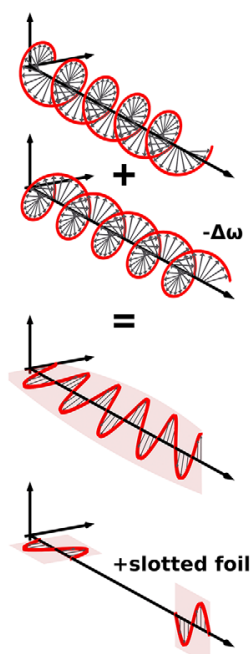

(c) combination of di erent frequencies
FIG. 2. Combination of radiation beams with different polarizations. Note that for illustration purposes the depicted rate of polarization change on subfigure (c) is exaggerated.

In general, one can modulate the phase difference $\Delta \phi$ as a function of time or transverse coordinate to shape the polarization of the output FEL radiation. The proposed schemes are illustrated on Figs. 3 and 4 respectively.

Also, the crossed undulator scheme relies upon an assumption that the radiation from the first undulator does not interact with the electron beam in the second, i.e., the radiation from the first undulator does not "seed" the second one.

In case of the crossed planar undulators this is always true, since the projection of the electric field of the "seed" onto the transverse electron velocity in the second undulator

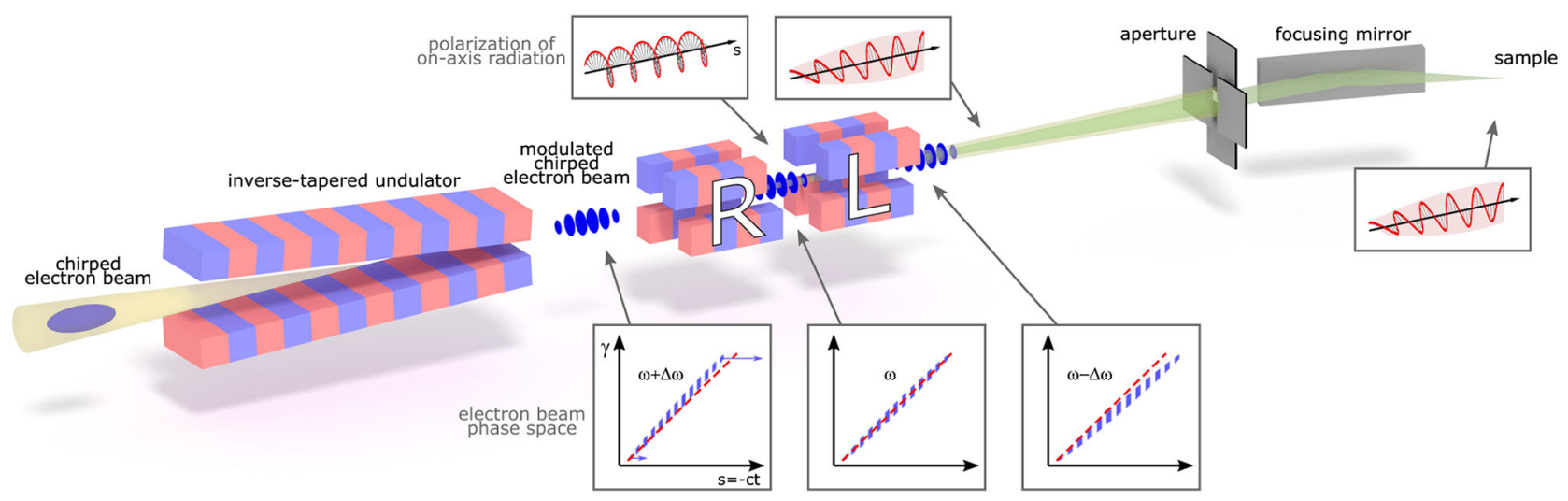

FIG. 3. Illustration of the scheme optimized for temporal polarization shaping or linearly polarized radiation. The density-modulated electron beam with a linear energy chirp sequentially radiates in the right- and left- handed helical undulators. Radiation frequency is determined by the bunching frequency, which changes upon passing through undulators. It is illustrated as "stretching" of the electron beam in comparison to a reference dashed line on the bottom inlays. This way one can obtain radiation with temporally shaped linear polarization. 


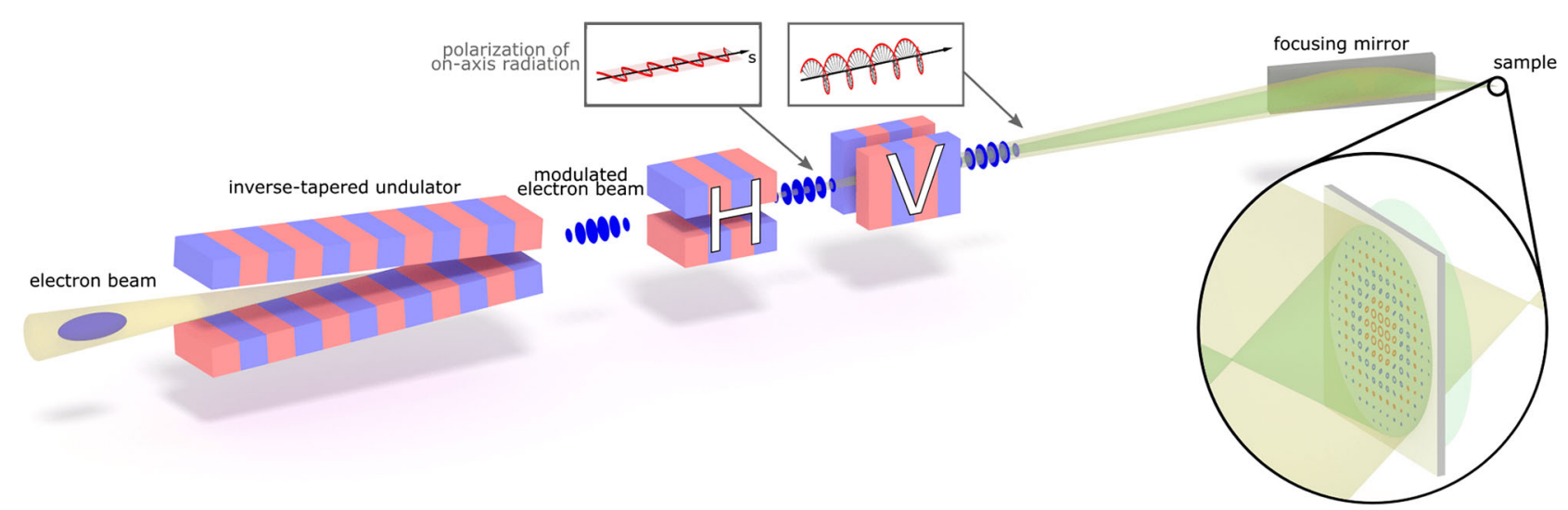

FIG. 4. Illustration of the scheme to deliver radiation with spatial polarization shaping. The density-modulated electron beam sequentially radiates in planar undulators set to horizontal and vertical polarization. After passing the imaging system depicted here as a single lens radiation is focused into two waists of orthogonal polarizations. The sample is introduced between the waists where spherical wavefronts of the two radiation beams have the opposite curvature. This results in spatially-dependent phase difference, hence spatially shaped polarization at the sample.

is always zero. In case of helical undulators set to orthogonal elliptical polarization basis this projection is not zero, but fluctuates along the undulator around zero value. Therefore, no synchronism takes place and on average the "seed" does not interact with the electron beam (see Appendix A).

\section{PROPOSED PRINCIPLE}

Consider two overlapped radiation pulses. The difference between their intensities and phases $\Delta \phi$ may be inhomogeneous either along the propagation direction (variation in time) or across the radial coordinate (variation in space) or both. Such variations cause degradation of the radiation polarization when averaged over time or across the transverse direction $[14,16]$.

Here we actually aim at maximizing this effect so that the resulting polarization may depend on the location on the sample and/or the arrival time of the radiation within the pulse. In order to illustrate this point, we use Stokes vector and Stokes angles formalism, revisited in Appendix B.

Let us consider the radiation distribution between the two identical FEL radiation sources modeled, for simplicity, as waists of Gaussian beams with sizes $w$, separated by distance $2 z_{u}$ and in right- and heft-handed circular polarization basis. Polarization properties between the waists are derived in Appendix C. Upon imaging them with a lens one expects to obtain the same polarization properties of the demagnified radiation, where temporal and spatial dependence of the Stokes angles is as follows:

$\psi(r, t, \Delta \omega)=-\frac{k r^{2}}{2 z_{u} M^{2}\left[1+\left(z_{R} / z_{u}\right)^{2}\right]}-\frac{\Delta \omega t}{2}+\phi_{0}$,

$\chi(r, t, \Delta \omega)=0$.

Here $\psi$ is the orientation angle of the polarization plane, $\chi$ is the deviation from purely linear polarization,
$z_{R}=k w^{2} / 2$ is the Rayleigh length of source Gaussian beams with wave vector $k=\omega / c . \omega$ and $\Delta \omega$ are the mean and difference of the beams carrier frequencies. $\phi_{0}$ is a phase difference between the two beams that does not depend on time or transverse coordinate and can be tuned with, for instance, a weak phase shifter. $M$ is the magnification of imaging system. One can see that the radiation is linearly polarized, with orientation of the polarization plane depending quadratically on a distance $r$ from the axis. This dependence, schematically illustrated on Fig. 5(a), is the example of transverse, or spatial polarization shaping. For comparison, Fig. 5(b) illustrates the radiation from two crossed planar undulators. This transverse polarization shaping effect is caused by different wavefront curvatures of the two superimposed radiation beams with equal intensities and different polarization states.

The rate of spatial polarization shaping, $d \psi / d r$ and/or $d \chi / d r$, can be controlled by varying distance between the

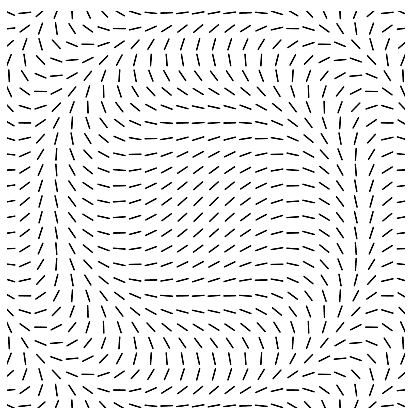

(a) sum of circularly polarized beams

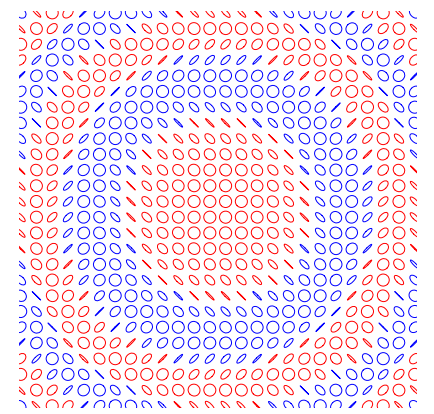

(b) sum of linearly polarized beams
FIG. 5. Illustration of spatial polarization shaping: polarization ellipses at different transverse coordinates arcoss the sample for crossed helical (left plot) and planar (right plot) undulators. Color depicts radiation helicity, e.g., red corresponds to right-handed polarization and blue to the left-handed one. 
image waists in terms of their Rayleigh lengths. The effect is maximized when $z_{u} \gg z_{R}$. Consequently, the spatial shaping may be minimized if the Rayleigh length of the images is increased by using a lens with a finite pupil while focusing on the sample.

From Eq. (1) it also follows that orientation of the polarization plane also varies with time at the rate of half the beam frequency difference $d \psi / d t=\Delta \omega / 2$. We refer to this effect as the longitudinal, or temporal polarization shaping. This effect is illustrated on Fig. 2(c).

In principle, pulse intensity can be modulated via slotted foil [17], laser heater [18], or fresh-slice technique [19,20] to facilitate pump-probe experiments. If the goal is to generate two distinct pulses with different polarizations, the proposed setup will be more limited compared to the method reported in [20]: it will yield less power, smaller delay range and will be harder to tune delay while keeping other parameters constant, photon energy is be fixed. However, in that case the proposed method would allow one to control the polarization of both pulses, while in [20] polarization of the first pulse is determined by the magnetic structure of the baseline planar undulator. In addition, source separation of the two pulses would be considerably smaller and the proposed scheme could allow phase stability between these pulses, provided the jitter in the electron beam is accounted for.

In practice, generation of two overlapped coherent FEL pulses with different frequencies creates a challenge that is discussed below.

\section{DETUNING FREQUENCY OF THE SECOND UNDULATOR RADIATION}

In order to generate the FEL radiation with temporally shaped polarization, the frequency of the electron beam density modulation (i.e., "bunching") should be shifted by $\Delta \omega$ between the ends of the two consequent radiators.

Such frequency shift takes place naturally in a dispersive environment with nonzero $R_{56}$ value, provided that the modulated electron beam has an energy chirp, as illustrated on Fig. 6. Here we remind that $R_{56}$ is related to the momentum compaction, and is an element of the transfer matrix of the setup. Its value between the undulator ends consists of that of a single undulator and an intersection:

$$
R_{56}=-\frac{1}{\gamma^{2}}\left[L_{u}\left(1+K^{2}\right)+L_{i s}\right]=-2 N_{u} \lambda-\frac{L_{i s}}{\gamma^{2}},
$$

where $K$ is the rms undulator parameter, $L_{u}$ and $L_{i s}$ are the undulator and intersection lengths correspondingly, $N_{u}$ denotes the number of undulator periods, and $\lambda=2 \pi c / \omega$ is the resonant undulator wavelength.

This dispersion is too small to strongly affect the density modulation amplitude, but is sufficient to change its frequency proportionally to the final electron beam elongation $\delta s=-R_{56} \Delta \gamma / \gamma$ :

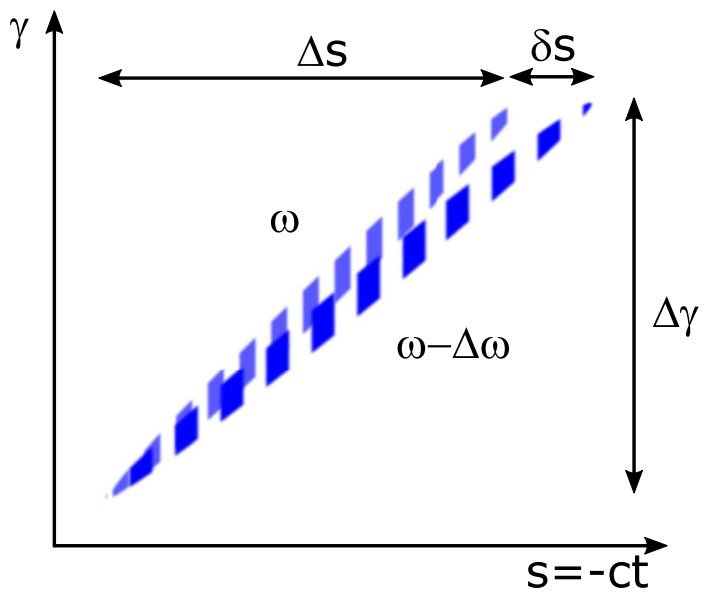

FIG. 6. Illustration of the longitudinal phase space of a linearly chirped electron beam with coherent density modulation at frequency $\omega$. This frequency is shifted by $\Delta \omega$ upon propagation through an element with non-zero compaction factor.

$$
\frac{\Delta \omega}{\omega}=-\frac{\delta s}{\Delta s}=\frac{R_{56} \Delta \gamma}{\gamma \Delta s} .
$$

The resulting shift of the bunching frequency $\Delta \omega$ weakly depends on the resonance frequency $\omega$ in the case of the short intersection and the large undulator parameter $K$ :

$$
\Delta \omega=-\left(\frac{\omega L_{i s}}{\gamma^{2}}+4 \pi c N_{u}\right) \frac{\Delta \gamma}{\gamma \Delta s} .
$$

The key element of the temporal polarization shaping is the energy chirp over the electron beam. If the temporal coherence of the bunching is poor (which is the case for SASE), the radiation emitted in the first radiator would slip forward considerably, compared to the SASE spike width (see Fig. 5 in [16]). Such slippage would yield undesired rapidly oscillating ratio of intensity of the two overlapped radiation pulses and consequently-unwanted oscillation of polarization state. Therefore, the temporal coherence should be maximized, however introducing a fully coherent seed is, strictly speaking, not necessary which potentially relaxes requirements to the seed laser or the self-seeding monochromator resolution. Similar reasoning can be applied to the seed laser phase noise as well as the intensity modulations, potentially caused by microbunching instability. The latter should be, if possible, minimized.

\section{PROPOSED SCHEME REALIZATION AND RESULTS OF NUMERICAL SIMULATIONS}

We exemplify the possibility of obtaining the FEL radiation with shaped polarization on the SASE3 undulator line of the European XFEL. Its essential parameters are provided in Table I. The main elements of the proposed setup are four helical APPLE-X radiator segments with full 
TABLE I. SASE3 undulator parameters.

\begin{tabular}{lc}
\hline \hline Parameter & Value \\
\hline$K_{\text {rms }}$ & $\leq 9$ \\
Period & $6.8 \mathrm{~cm}$ \\
Undulator length & $5 \mathrm{~m}$ \\
Intersection length & $1.1 \mathrm{~m}$ \\
No. of segments & 21 \\
\hline \hline
\end{tabular}

TABLE II. Electron beam parameters.

\begin{tabular}{lc}
\hline \hline Parameter & Value \\
\hline Energy $\gamma$ & 16600 \\
Energy chirp $\Delta \gamma / \Delta s$ & $0 ; 5 / \mu \mathrm{m}$ \\
Energy spread $\sigma_{\gamma}$ & 3.5 \\
Norm. emittance & $0.5 \mathrm{~mm} \cdot \mathrm{mrad}$ \\
$\beta$ (average) & $20 \mathrm{~m}$ \\
\hline \hline
\end{tabular}

polarization control, proposed for installation at the facility [9] and composed of twenty-two $9 \mathrm{~cm}$-long periods each. For the sake of argument, here we will conventionally assume that the first radiator is tuned to emit radiation with right-handed circular polarization as defined from the point of view of a downstream observer (negative helicity), while the second one produces left-handed circularly polarized radiation (positive helicity). The considered photon energy is $300 \mathrm{eV}$.

The parameters of the electron beam used for simulations are presented on Table II. We assume that the electron beam with coherent density modulation can be generated using a combination of external seeding or self-seeding technique [21-23] and inverse tapering [24,25]. We model the FEL performance with the numerical code Genesis [26] and analyze radiation properties with Ocelot [27].

\section{A. Scheme optimized for temporal polarization shaping}

The scheme to generate temporally shaped FEL radiation is depicted on Fig. 3. An electron beam with uniform properties except for a linear energy chirp is overlapped with coherent radiation pulse-seed-as depicted on Fig. 7(a), and introduced in the nominal SASE3 undulator. The electron beam energy chirp can be controlled by modifying electron beam compression and/or using longitudinal wakefield structure, while keeping in mind possible chirp nonlinearities.

The rate of the temporal polarization shaping is determined by the slope of linear chirp along the electron beam. The deviations from strictly linear chirp would cause variation of rate of temporal polarization shaping. The chirp should be at least diagnosed with, for example, a transverse deflection cavity, and its quadratic component within the lasing window should be minimized.

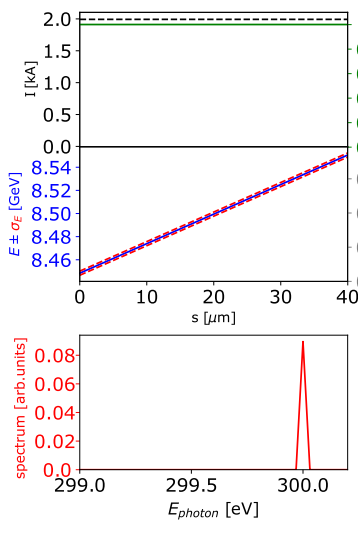

(a) modulator entrance
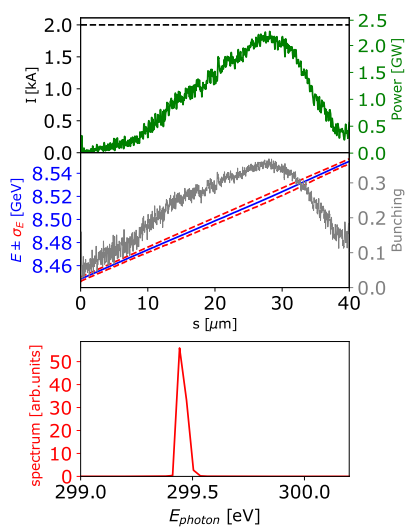

(c) $1^{\text {st }}$ radiator end, right-handed polarization
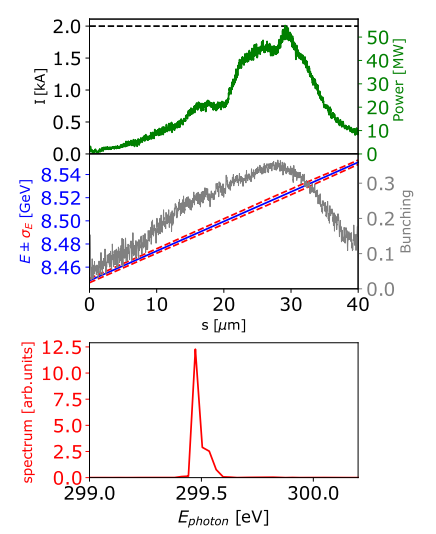

(b) modulator end
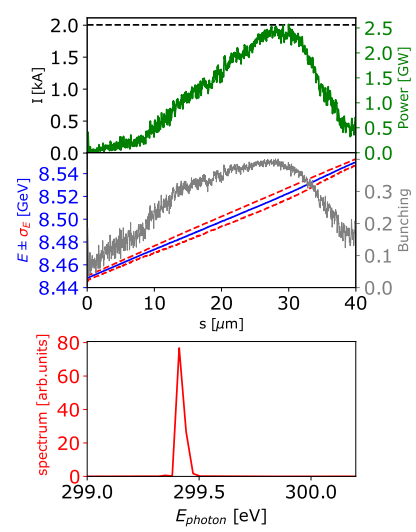

(d) $2^{\text {nd }}$ radiator end, left-handed polarization
FIG. 7. Properties of electron beam and FEL radiation at different locations along the undulator. Top subplots depict the electron beam current (black dached line, $2 \mathrm{kA}$ constant), and radiation power (green solid line). Middle subplots depict mean (blue line) and $2 \sigma$ spread (red dashed lines) of the electron beam energy as well as its bunching (grey line). Bottom plots show the on-axis spectra of the radiation.

Undulator inverse tapering [28,29] allows to amplify electron bunching [see Fig. 8(a)] while keeping emitted radiation at relatively low level, as depicted on Fig. 7(b). Therefore one can refer to this inverse tapered undulator as modulator.

Bunching in the electron beam is not uniformly high at the end of the modulator, since the interaction between the monochromatic seed and energy-chirped electron beam cannot take place well beyond the undulator resonance condition. This interaction window along the electron beam is in order of $\rho \gamma \Delta s / \Delta \gamma$ and defines the upper limit of the FEL pulse length with temporal polarization shaping. Here $\rho$ is the FEL gain (Pierce) parameter that determines the amplification resonance bandwidth. The interaction window can be increased by applying frequency chirp to the seed radiation [30], however it may increase the complexity of the method and subsequent data analysis. 


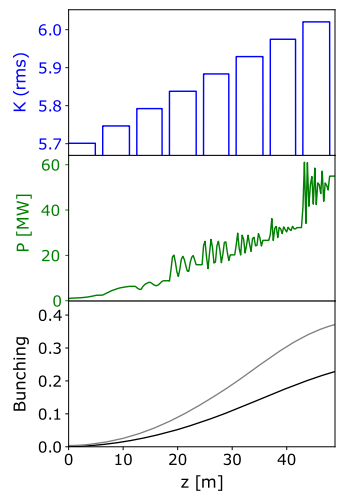

(a) modulator

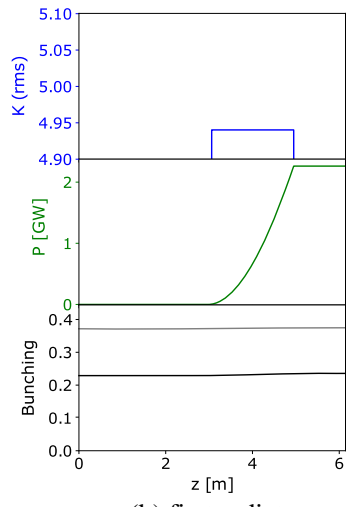

(b) first radiator

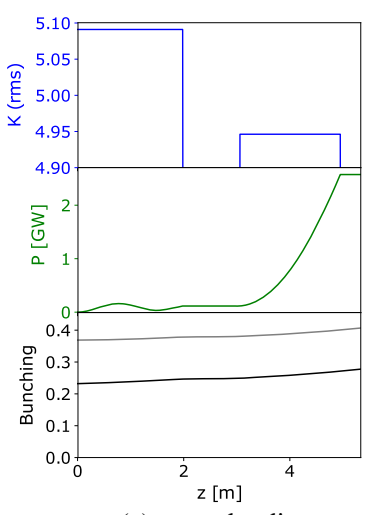

(c) second radiator
FIG. 8. Growth of the radiation power and the electron beam bunching (grey line-peak value, black line-current-weighted average) as a function of the undulator length.

The modulated electron beam is fed sequentially through four helical radiator segments. The first radiator segment is effectively extracted. The second one is resonant to the bunching frequency and emits right-handed circular polarization. In simulation both radiation and electron distribution files are dumped, the latter is used as an input for the following simulation stage. Third helical undulator is detuned beyond the FEL resonance but contributes to the shift of bunching frequency due to its compaction factor. Fourth is resonant to the slightly shifted bunching frequency and is set to emit radiation with left-handed circular polarization. Distance between centers of the resonant radiators is $6.2 \mathrm{~m}$. As a result, two radiation pulses with comparable radiation power levels of $2 \mathrm{GW}$ and $0.03 \mathrm{eV}$ photon energy separation are generated, as shown on last subfigures of Figs. 7 and 8.

We filtered high angular frequencies of the emitted radiation to imitate a square aperture with a size of $20 \mathrm{~mm}$ or $10 \mathrm{~mm}$ at a distance of $500 \mathrm{~m}$ from the radiators. By varying the aperture size one can reach a desired tradeoff between its transmission (here $48 \%$ and $12 \%$ ) and the transversely averaged degree of polarization $(75 \%$ and $98 \%$ correspondingly).

We assume that the distance between the focusing mirror and the sample is $2.5 \mathrm{~m}$ and the mirror is located next to the
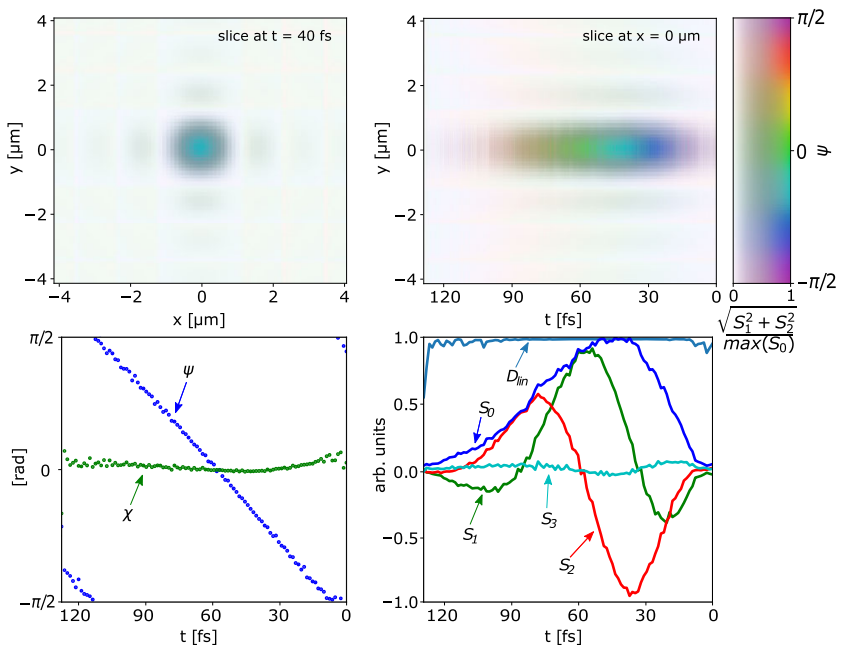

FIG. 9. Radiation polarization properties for the setup geometry optimized for temporal polarization shaping. Stokes parameters averaged over 1.3 fs (19 simulation slices). Here we assume $10 \mathrm{~mm}$ aperture size. Top plots depict radiation intensity and orientation of the polarization plane in false colors as a function of transverse coordinates and time. Bottom left plot depicts angles of Stokes vector averaged over transverse coordinates as a function of time. Bottom right plot depicts the corresponding Stokes vector components.

aperture. Therefore we scale the transverse mesh down by factor $M=1 / 200$ to account for the mirror demagnification. This allows us to provide good estimation of the radiation spot size and polarization distribution at the sample. The Stokes parameters of this imaged radiation with temporal polarization shaping for $10 \mathrm{~mm}$ aperture are provided in Fig. 9.

For simplicity, we assumed a single ellipsoidal mirror as focusing element. In practice, Kirkpatrick-Baez mirrors may be used to carry out the focusing. Such setup would yield magnification ratio for vertical and horizontal planes as large as $2: 1$. In that case the distribution presented on the figures would be asymmetric by the same factor.

Rapid temporal polarization shaping is also referred to as "polarization scrambling." From Fig. 9 it follows that the frequency of this scrambling with the proposed scheme may reach several teraradians per second.

\section{B. Scheme optimized for spatial polarization shaping}

In order to generate FEL radiation with strong spatial polarization shaping, the setup, depicted on Fig. 4 is principally the same, except there is no need for the electron energy chirp, and distance between the active helical radiators should be maximized. In our case the source separation of $9.2 \mathrm{~m}$ is larger than the equivalent of Rayleigh length $\left(z_{R} \sim 2 \mathrm{~m}\right)$. Also, no aperture at the lens position is needed, on the contrary: radiation clipping by the lens edges is undesired. In contrast to the temporal shaping, spatial shaping imposes no limitations on resulting pulse duration. 

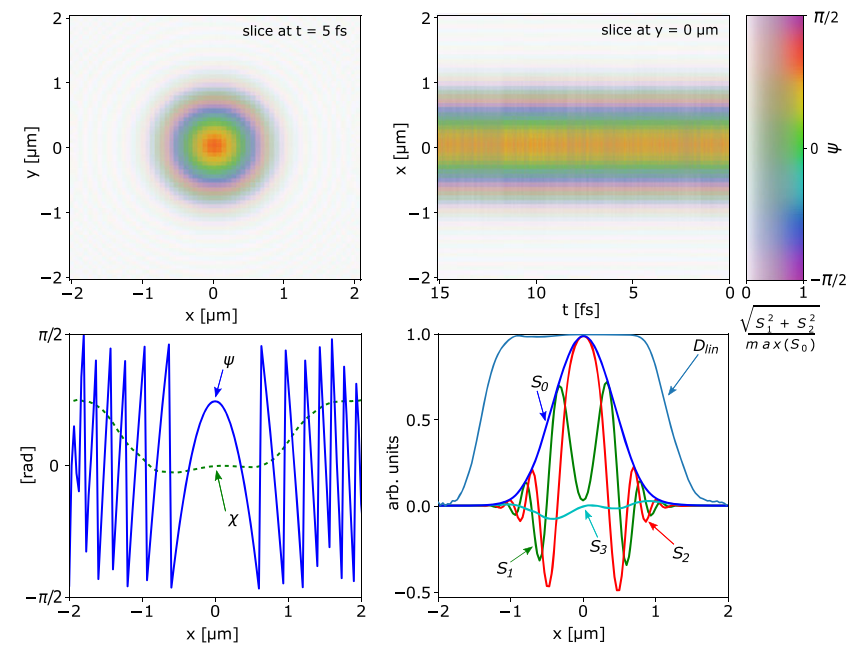

FIG. 10. Polarization properties of radiation for the setup geometry optimized for spatial polarization shaping with crossed helical undulators. Stokes parameters averaged over 15 fs. Notation is the same as in Fig. 9, except the Stokes vector and angles are calculated for the radiation at slice $y=0$ and averaged over time. Radiation power is $3 \mathrm{GW}$.

Stokes parameters of such radiation at the sample location are provided in Figs. 10 and 11, illustrating polarization properties of crossed helical and planar undulators correspondingly.

Spatial polarization shaping of the wavefront may enable single shot studies of dichroic materials at $\mathrm{x}$-ray absorption resonances. When propagating through magnetic or chiral materials the wavefront is modulated which can be captured on an array detector. In time-resolved studies this would offer the possibility to record nonrepeatable dynamics when both polarization components are simultaneously
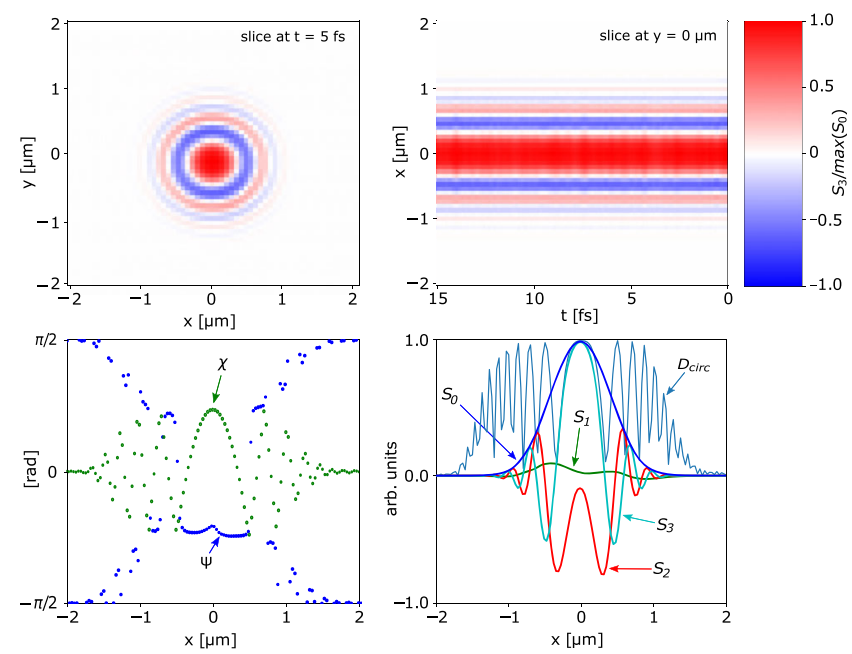

FIG. 11. Polarization properties of radiation for the setup geometry optimized for spatial polarization shaping with crossed planar undulators. Notation and averaging are the same as in Fig. 10. Radiation power is $1.5 \mathrm{GW}$. required for signal detection [31]. In a two-color excitation scheme in case that the experimental conditions enable to extract spatially or temporally resolved pump-probe overlap signals more rich information, up to the full picture of the underlying dynamics on the femtosecond timescale, can be obtained from a single pulse measurement [32].

\section{CONCLUSIONS}

In this paper we presented a cost-effective method to shape the polarization state of seeded FEL radiation pulses impinging on the sample as a function of time and/or transverse coordinate.

In order to generate radiation with shaped polarization, two distinct temporally coherent radiation pulses with orthogonal polarization states should be emitted in two consecutive radiators by a coherently modulated electron beam.

Polarization can be shaped temporally at the rate of half the radiation pulses frequency difference. This rate is proportional to magnitude of the linear energy chirp in the electron beam. We have found that it should be possible to "scramble" the polarization of the FEL radiation with rate of several Tera-radians per second. A few short pulses with polarization-of-interest may be selected by modulating the FEL power along the pulse by, for example, introducing a slotted foil in the accelerator.

Polarization can also be modulated spatially if the wavefronts of the overlapped pulses have different curvatures. This effect can be either maximized if the radiators are separated by more than several Rayleigh lengths or mitigated by filtering the radiation with an aperture in the far zone.

We confirm and exemplify our findings with results of numerical FEL simulations for SASE3 undulator line at the European XFEL. Also we believe that this paper will contribute to development of experimental techniques utilizing complex polarized light and advanced schemes for its generation.

\section{ACKNOWLEDGMENTS}

We would like to thank Andreas Scherz, Evgeny Saldin, Takanori Tanikawa, Sergey Tomin, Suren Karabekyan, Michael Meyer for useful discussions and Serguei Molodtsov for his interest in this work.

\section{APPENDIX A: RESONANT INTERACTION}

The rate of energy exchange between an electron and a radiation field propagating in the same direction is given by:

$$
\frac{d \varepsilon}{d t} \sim \boldsymbol{V}_{\perp} \cdot \boldsymbol{E}=V_{x} E_{x}+V_{y} E_{y},
$$

where $V_{\perp}$ is the transverse component of the electron velocity. If the interaction takes place in a planar undulator where the electron undulates in the horizontal plane, while 
the radiation field is polarized in the vertical direction, one always has

$$
\frac{d \varepsilon}{d t} \sim V_{x} \cdot 0+0 \cdot E_{y}=0 .
$$

Therefore, in an FEL such radiation would not contribute to the FEL amplification process. In other words, it is impossible to "seed" the FEL with radiation of orthogonal polarization.

Let us make an extension to the helical undulator case and circularly polarized radiation with a field given by:

$$
\begin{aligned}
\boldsymbol{E} & =E_{x} \boldsymbol{e}_{x}+E_{y} \boldsymbol{e}_{y}, \\
E_{x} & =E_{0} \cos (k z-\omega t+\phi), \\
E_{y} & =E_{0} \sin (k z-\omega t+\phi),
\end{aligned}
$$

which is overlapped with the electron in a helical undulator with velocity

$$
\begin{aligned}
& \boldsymbol{V}_{\perp}=V_{x} \boldsymbol{e}_{x}+V_{y} \boldsymbol{e}_{y}, \\
& V_{x}=+\frac{K c}{\gamma} \cos \left(k_{w} z\right), \\
& V_{y}= \pm \frac{K c}{\gamma} \sin \left(k_{w} z\right) .
\end{aligned}
$$

Depending on the undulator helicity, the electron energy transfer rate is

$$
\begin{aligned}
& \frac{d \varepsilon}{d t} \sim V_{x} E_{x}+V_{y} E_{y} \\
& =\frac{K c E_{0}}{\gamma}\left[\cos \left(k_{w} z\right) \cos (k z-\omega t+\phi)\right. \\
& \left. \pm \sin \left(k_{w} z\right) \sin (k z-\omega t+\phi)\right],
\end{aligned}
$$

so

$$
\frac{d \varepsilon}{d t} \sim \cos \left(\mp k_{w} z+k z-\omega t+\phi\right) .
$$

We can redefine the argument of cosine as a phase $\psi=$ $\left(\mp k_{w}+k\right) z-\omega t+\phi$. The effective energy exchange between the electron and the radiation take place if $\psi$ is nearly constant along the undulator length:

$$
\frac{d \psi}{d t}=\left(\mp k_{w}+k\right) V_{z}-c k,
$$

where $V_{z}=c\left(1-\frac{1+K^{2}}{2 \gamma^{2}}\right)$. Using it one obtains:

$$
\frac{d \psi}{d t}=c k\left(\mp \frac{k_{w}}{k}-\frac{1+K^{2}}{2 \gamma^{2}}\right) .
$$

The resonance condition is fulfilled if one takes the plus sign but it is impossible to fulfill $d \psi / d t=0$ if the sign before $k_{w} / k$ is negative (radiation polarization is opposite to the undulator helicity). The phase $\psi$ is rapidly oscillating along the undulator, and, on average, along the undulator no energy transfer between electrons and radiation takes place.

Our reasoning can be applied to a general case of elliptically polarized radiation propagating in an undulator with elliptical helicity. The field is given in the form:

$$
\begin{aligned}
\boldsymbol{E} & =E_{x} \boldsymbol{e}_{x}+E_{y} \boldsymbol{e}_{y}, \\
E_{x} & =E_{0 x} \cos \left(k z-\omega t+\chi_{1}+\phi\right), \\
E_{y} & =E_{0 y} \sin (k z-\omega t+\phi),
\end{aligned}
$$

and the expression for velocity is following:

$$
\begin{aligned}
\boldsymbol{V}_{\perp} & =V_{x} \boldsymbol{e}_{x}+V_{y} \boldsymbol{e}_{y}, \\
V_{x} & =\frac{K_{x} c}{\gamma} \cos \left(k_{w} z+\chi_{2}\right) \\
V_{y} & =\frac{K_{y} c}{\gamma} \sin \left(k_{w} z\right) .
\end{aligned}
$$

These expressions for the field and velocity are the parametric equations of two ellipses with a free variable $z$ and $t$ which is fixed. The phases $\chi_{1}$ and $\chi_{2}$ along with ratios $E_{0 x} / E_{0 y}$ and $K_{x} / K_{y}$ define parameters of the ellipses and the phase $\phi$ sets an arbitrary phase shift between the electric field and electron trajectory.

Thus, the energy transfer rate along the $z$ axis is defined by the scalar product of the field and velocity:

$$
\begin{aligned}
\frac{d \varepsilon}{d z} \sim & K_{x} E_{0 x} \cos \left(k_{w} z+\chi_{2}\right) \cos \left(k z-\omega t+\chi_{1}+\phi\right) \\
& +K_{y} E_{0 y} \sin \left(k_{w} z\right) \sin (k z-\omega t+\phi) .
\end{aligned}
$$

Assuming $K_{x} E_{0 x}=K_{y} E_{0 y}$ and making some simplifications we have:

$$
\begin{aligned}
\frac{d \varepsilon}{d z} \sim & \cos \left(-k_{w} z+k z-\omega t+\chi_{1}-\chi_{2}+\phi\right) \\
& +\cos \left(+k_{w} z+k z-\omega t+\chi_{1}+\chi_{2}+\phi\right) \\
& +\cos \left(-k_{w} z+k z-\omega t+\phi\right) \\
& -\cos \left(+k_{w} z+k z-\omega t+\phi\right) .
\end{aligned}
$$

To average the interaction to zero we need to set $\chi_{1}=-\chi_{2}$. Mathematically, the relations $K_{x} E_{0 x}=K_{y} E_{0 y}$ (or $K_{x} / K_{y}=$ $\left.E_{0 y} / E_{0 x}\right)$ and $\chi_{1}=-\chi_{2}$ define two ellipses with the same eccentricity and orthogonal orientation of axes. On Fig. 12 we depict variation of two such ellipses with respect to the phase $\chi_{1}$. In terms of physics, the condition $K_{x} E_{0 x}=$ $K_{y} E_{0 y}$ shows that the energy transfer is distributed equally between $x$ and $y$ axes.

Finally, it is shown that there is zero average energy transfer for the more general case of an elliptically polarized radiation and the electron moving with the opposite helicity. 


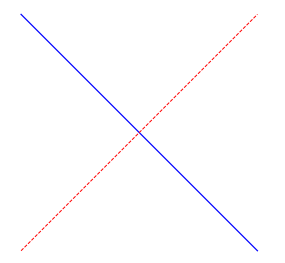

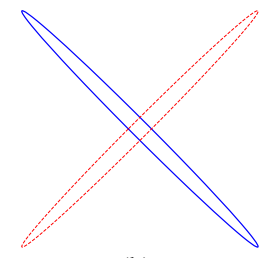

(b)

(a) $\chi_{1}=\pi / 2$

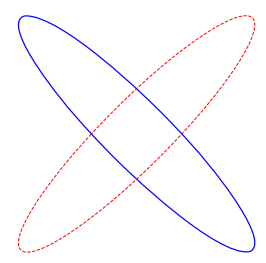

(d)

$\chi_{1}=\pi / 2+\pi / 8$ $\chi_{1}=\pi / 2+\pi / 32$

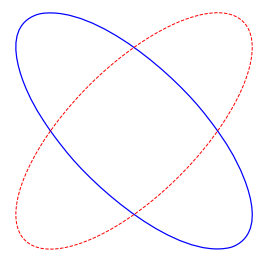

(e)

$\chi_{1}=\pi / 2+\pi / 4$

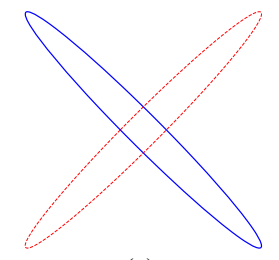

(c) $\chi_{1}=\pi / 2+\pi / 16$

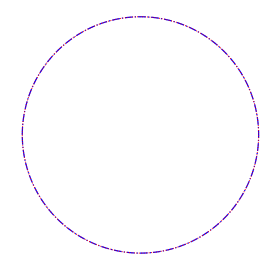

(f)
FIG. 12. Hodograph of elliptical polarization state (blue line) and velocity in a helical undulator (red line) depending on the phase $\chi_{1}$.

\section{APPENDIX B: STOKES PARAMETERS}

Consider a uniform, well-collimated, quasimonochromatic light beam with mean frequency $\omega$, propagating in direction $z$. The transverse component of the electric field vector can be decomposed into either two orthogonal components along the $x$ - and $y$-basis

$$
\boldsymbol{E}(x, y, z ; t)=E_{x}(x, y, z ; t) \boldsymbol{e}_{x}+E_{y}(x, y, z ; t) \boldsymbol{e}_{y},
$$

as well as into circular basis

$$
\boldsymbol{E}(x, y, z ; t)=E_{R}(x, y, z ; t) \boldsymbol{e}_{R}+E_{L}(x, y, z ; t) \boldsymbol{e}_{L} .
$$

We consider here the field $\boldsymbol{E}$ as a complex value associated with the real-valued electric field. Relation between Cartesian basis and circular basis is given here:

$$
\begin{aligned}
& \boldsymbol{e}_{R}=\frac{1}{\sqrt{2}}\left(\boldsymbol{e}_{x}+i \boldsymbol{e}_{y}\right) \\
& \boldsymbol{e}_{L}=\frac{1}{\sqrt{2}}\left(\boldsymbol{e}_{x}-i \boldsymbol{e}_{y}\right) .
\end{aligned}
$$

It is convenient to describe the polarization state of this quasimonochrmatic radiation with Stokes parameters:

$$
\begin{aligned}
& S_{0}(x, y, z ; t ; \Delta t)=\left\langle E_{x} E_{x}^{*}\right\rangle+\left\langle E_{y} E_{y}^{*}\right\rangle \\
& S_{1}(x, y, z ; t ; \Delta t)=\left\langle E_{x} E_{x}^{*}\right\rangle-\left\langle E_{y} E_{y}^{*}\right\rangle \\
& S_{2}(x, y, z ; t ; \Delta t)=\left\langle E_{y} E_{x}^{*}\right\rangle+\left\langle E_{x} E_{y}^{*}\right\rangle \\
& S_{3}(x, y, z ; t ; \Delta t)=i\left(\left\langle E_{y} E_{x}^{*}\right\rangle-\left\langle E_{x} E_{y}^{*}\right\rangle\right),
\end{aligned}
$$

where $\langle\ldots\rangle$ denote the average over the observation time $\Delta t$. In the circular basis the Stokes parameters are defined in the following way:

$$
\begin{aligned}
& S_{0}(x, y, z ; t ; \Delta t)=\left\langle E_{R} E_{R}^{*}\right\rangle+\left\langle E_{L} E_{L}^{*}\right\rangle \\
& S_{1}(x, y, z ; t ; \Delta t)=\left\langle E_{R} E_{L}^{*}\right\rangle+\left\langle E_{L} E_{R}^{*}\right\rangle \\
& S_{2}(x, y, z ; t ; \Delta t)=i\left(\left\langle E_{R} E_{L}^{*}\right\rangle-\left\langle E_{L} E_{R}^{*}\right\rangle\right) \\
& S_{3}(x, y, z ; t ; \Delta t)=\left\langle E_{L} E_{L}^{*}\right\rangle-\left\langle E_{R} E_{R}^{*}\right\rangle .
\end{aligned}
$$

The Stokes vector [33] $\boldsymbol{S}(x, y, z ; t ; \Delta t)=\left(S_{1}, S_{2}, S_{3}\right)$ characterizes the radiation polarization upon averaging over the time interval $\Delta t$. The polarization of the radiation can also be represented in terms of the azimuthal and polar angles $\psi$ and $\chi$ of the Stokes vector in spherical coordinate system:

$$
\begin{gathered}
2 \psi(s, \Delta t)=\arctan \left(S_{2} / S_{1}\right), \\
2 \chi(s, \Delta t)=\arctan \left(S_{3} / \sqrt{S_{1}^{2}+S_{2}^{2}}\right) .
\end{gathered}
$$

Here the orientation of the polarization plane is defined by the angle $2 \psi$, whereas the deviation from pure linear polarization is determined by the angle $2 \chi$. In order to visualize the polarization state one can use the Poincaré sphere or the polarization ellipse, both illustrated on Fig. 13.

The physical meaning of the Stokes parameter $S_{0}$ is the total radiation power $P_{\text {tot }}$, averaged over a measurement time $\Delta t$. The $S_{1}$, as it can be seen from the definition in Cartesian basis, indicates the difference between the intensities of light polarized along the $x$-axis and $y$-axis respectively. The same for $S_{2}$ but for the polarization along the directions $45^{\circ}$ and $135^{\circ}$ that are measured with respect the $x$-axis. $S_{3}$ has the meaning of the difference between L-handed and R-handed polarization. So, $S_{1}$ and $S_{2}$ show the properties of linearly polarized light and $S_{3}$ indicates any deviation from linearity.

We can interpret the length of the Stokes vector $\boldsymbol{S}(x, y, z ; t ; \Delta t)=\left(S_{1}, S_{2}, S_{3}\right)$ as an effective intensity of the polarized fraction of the radiation $P_{\mathrm{pol}}$ :

$$
P_{\mathrm{pol}}=|\boldsymbol{S}|=\sqrt{S_{1}^{2}+S_{2}^{2}+S_{3}^{3}} \leq S_{0} .
$$

Then, the degree of polarization is given by
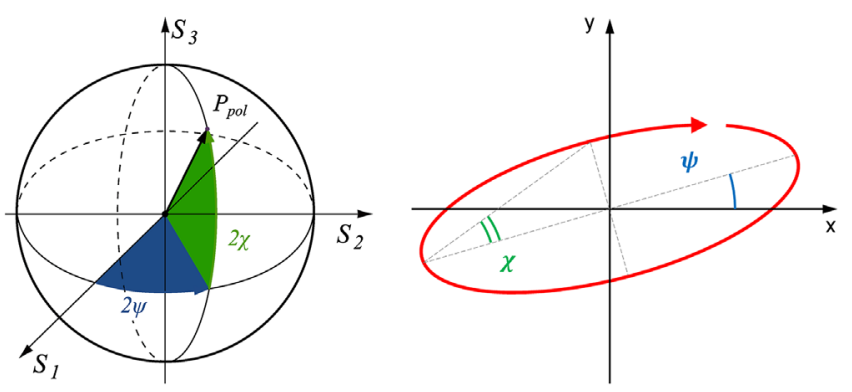

FIG. 13. Poincaré sphere and polarization ellipse. 


$$
D_{\mathrm{pol}}(s, \Delta t)=\frac{P_{\mathrm{pol}}}{P_{\mathrm{tot}}}=\frac{\sqrt{S_{1}^{2}+S_{2}^{2}+S_{3}^{2}}}{S_{0}} .
$$

The effective power of the linearly polarized portion of the radiation corresponds to the projection of the Stokes vector $S$ onto the $\left(S_{1} S_{2}\right)$ plane:

$$
P_{\text {lin pol }}=\sqrt{S_{1}^{2}+S_{2}^{2}}
$$

and the corresponding degrees of linear and circular polarizations are

$$
\begin{gathered}
D_{\text {lin pol }}(s, \Delta t)=\frac{P_{\text {lin pol }}}{P_{\text {tot }}}=\frac{\sqrt{S_{1}^{2}+S_{2}^{2}}}{S_{0}}, \\
D_{\text {circ pol }}(s, \Delta t)=\frac{P_{\text {circ pol }}}{P_{\text {tot }}}=\frac{\left|S_{3}\right|}{S_{0}} .
\end{gathered}
$$

Amplitudes and phases of the time varying complex amplitude $\tilde{\boldsymbol{E}}$ of the quasimonochromatic electric field $\boldsymbol{E}=\tilde{\boldsymbol{E}} \exp \left(i \omega_{c} z / c\right)$ are assumed to vary slowly along $z$ direction, in comparison to the carrier wavelength $\lambda_{c}=\omega_{c} / c$. We can introduce the characteristic time of this variation $\tau \gg 2 \pi / \omega_{c}$. In this case, the radiation may be considered polarized on timescales $2 \pi / \omega_{c} \ll \Delta t \ll$ $\tau$ and unpolarized on timescales $\Delta t \gg \tau$, given that $D_{\text {pol }}(\Delta t)=0$.

\section{APPENDIX C: DERIVATION OF STOKES PARAMETERS FOR TWO HELICAL UNDULATORS}

In order to model an FEL radiation we use the Gaussian beam formalism. The radiation field at distance $z$ from the Gaussian beam waist is given by:

$$
\begin{aligned}
& \boldsymbol{E}(x, y, z ; t)=A \boldsymbol{e}_{1} \frac{w_{0}}{w(z)} \exp [i \phi(z)+i k z-i \omega t] . \\
& \exp \left[-\frac{x^{2}+y^{2}}{w^{2}(z)}\right] \exp \left[i \frac{k}{2 R(z)}\left(x^{2}+y^{2}\right)\right]
\end{aligned}
$$

where $\boldsymbol{e}_{1}$ denotes for an arbitrary polarization state,

$$
\begin{gathered}
w^{2}(z)=w_{0}^{2}\left[1+\left(z / z_{R}\right)^{2}\right], \\
R(z)=z\left[1+\left(z_{R} / z\right)^{2}\right],
\end{gathered}
$$

and the Gouy phase shift $\phi(z)$ is

$$
\phi(z)=-\arctan \left[\frac{z}{z_{R}}\right] .
$$

Light of the two Gaussian beams can be propagated by the distance $z_{u}$ and $-z_{u}$ accordingly to imitate the radiation distribution between the undulators. We assume that the radiation is circularly polarized with equal intensity, opposite helicity and carrier frequencies different by $\Delta \omega$. The field given by $(\mathrm{C} 1)$ contains the following functions of $z$ : $w(z), R(z)$, and $\phi(z)$. Here $w(z)$ is an even function so $w(-z)=w(z) \cdot R(z)$ and $\phi(z)$ are odd.

The expression for the fields becomes

$$
\begin{aligned}
E_{L}\left(x, y, z_{u} ; t\right)= & A \frac{w_{0}}{w\left(z_{u}\right)} \exp \left[i \phi\left(z_{u}\right)+i k z_{u}-i \omega t\right] . \\
& \exp \left[-\frac{x^{2}+y^{2}}{w^{2}\left(z_{u}\right)}\right] \exp \left[i \frac{k}{2 R\left(z_{u}\right)}\left(x^{2}+y^{2}\right)\right], \\
E_{R}\left(x, y,-z_{u} ; t\right)= & A \frac{w_{0}}{w\left(z_{u}\right)} \exp \left[-i \phi\left(z_{u}\right)-i k z_{u}\right. \\
& -i(\omega-\Delta \omega) t] \exp \left[-\frac{x^{2}+y^{2}}{w^{2}\left(z_{u}\right)}\right] \\
& \times \exp \left[i \frac{k}{-2 R\left(z_{u}\right)}\left(x^{2}+y^{2}\right)\right] .
\end{aligned}
$$

The expressions for the $E_{R} E_{R}^{*}, E_{L} E_{L}^{*}, E_{R} E_{L}^{*}, E_{L} E_{R}^{*}$ can be written as:

$$
\begin{aligned}
& E_{R} E_{R}^{*}= E_{L} E_{L}^{*}=\left(\frac{A w_{0}}{w\left(z_{u}\right)}\right)^{2} \exp \left[-\frac{2\left(x^{2}+y^{2}\right)}{w^{2}\left(z_{u}\right)}\right], \\
& E_{R} E_{L}^{*}=\left(E_{L} E_{R}^{*}\right)^{*}=\left(\frac{A w_{0}}{w\left(z_{u}\right)}\right)^{2} \exp \left[-\frac{2\left(x^{2}+y^{2}\right)}{w^{2}\left(z_{u}\right)}\right] . \\
& \quad \exp \left[-i \frac{k}{R(z)}\left(x^{2}+y^{2}\right)+2 i \phi(z)+2 i k z_{u}+i \Delta \omega t\right] .
\end{aligned}
$$

Using the Euler's formula $e^{i \phi}=\cos \phi+i \sin \phi$ and taking into account

$$
\begin{aligned}
& E_{R} E_{L}^{*}+\left(E_{L} E_{R}^{*}\right)^{*}=2\left|E_{R} E_{L}^{*}\right| \cos \left[\operatorname{Arg}\left(E_{R} E_{L}^{*}\right)\right] \\
& E_{R} E_{L}^{*}-\left(E_{L} E_{R}^{*}\right)^{*}=2 i\left|E_{R} E_{L}^{*}\right| \sin \left[\operatorname{Arg}\left(E_{R} E_{L}^{*}\right)\right]
\end{aligned}
$$

we can extract the Stokes parameters of the field between the waists of Gaussian beams:

$$
\begin{aligned}
& S_{0}(x, y, t, \Delta \omega)=2\left(\frac{A w_{0}}{w\left(z_{u}\right)}\right)^{2} \exp \left[-\frac{2\left(x^{2}+y^{2}\right)}{w^{2}\left(z_{u}\right)}\right] \\
& S_{1}(x, y, t, \Delta \omega)=2\left(\frac{A w_{0}}{w\left(z_{u}\right)}\right)^{2} \exp \left[-\frac{2\left(x^{2}+y^{2}\right)}{w^{2}\left(z_{u}\right)}\right] . \\
& \cos \left(2 \phi\left(z_{u}\right)+2 k z_{u}-\frac{k}{R\left(z_{u}\right)}\left(x^{2}+y^{2}\right)+\Delta \omega t\right) \\
& S_{2}(x, y, t, \Delta \omega)=-2\left(\frac{A w_{0}}{w\left(z_{u}\right)}\right)^{2} \exp \left[-\frac{2\left(x^{2}+y^{2}\right)}{w^{2}\left(z_{u}\right)}\right] . \\
& \sin \left(2 \phi\left(z_{u}\right)+2 k z_{u}-\frac{k}{R\left(z_{u}\right)}\left(x^{2}+y^{2}\right)+\Delta \omega t\right) \\
& S_{3}(x, y, t, \Delta \omega)=0 .
\end{aligned}
$$

For the Stokes angle $\psi$ it is necessary to take into account periodicity of the $\arctan (\tan ())$ function, so the resulting Stokes angles are 


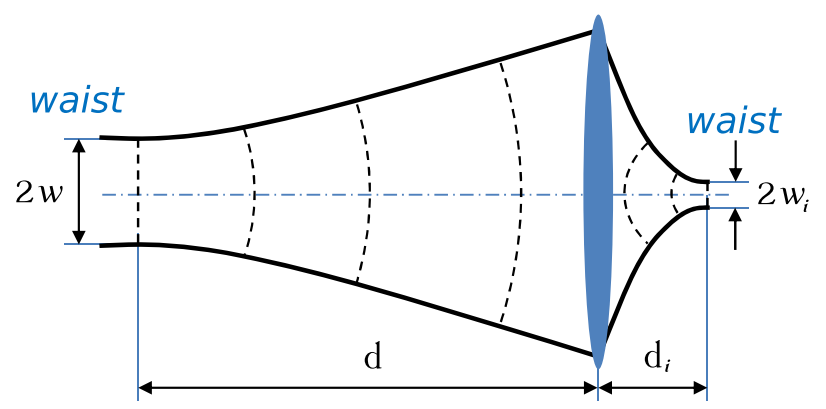

FIG. 14. Gaussian beam transformation by a lens. A Gaussian beam with plane wavefront and waist $w_{s}$, located at a distance $d_{s}$ from the thin lens with focal length $f$ is transformed to a Gaussian beam of plane wavefront and waist $w_{i}$, located at a distance $d_{i}$.

$$
\begin{aligned}
& \psi(x, y, t, \Delta \omega)=\phi\left(z_{u}\right)+k z_{u}-\frac{k r^{2}}{2 z_{u}\left[1+\left(z_{R} / z_{u}\right)^{2}\right]}+\frac{\Delta \omega t}{2}, \\
& \chi(x, y, t, \Delta \omega)=0 .
\end{aligned}
$$

Each of the radiation beams in Eq. (C5) can be imaged with a lens at distance $d_{s}$ from the source waist. Size of the image of the waist $w_{i}$, its Rayleigh length and distance from the lens $d_{i}$ would be

$$
\begin{gathered}
w_{i}^{2}=\frac{w^{2} f^{2}}{\left[\left(d_{s}-f\right)^{2}+z_{R s}^{2}\right]}, \\
d_{i}=f+f^{2} \frac{\left(d_{s}-f\right)}{\left[\left(d_{s}-f\right)^{2}+z_{R s}^{2}\right]},
\end{gathered}
$$

following the notation in Fig. 14. Given the magnification factor of such imaging system,

$$
M=\frac{d_{s}}{d_{i}}=\frac{f}{d_{s}-f},
$$

waist sizes, their Rayleigh lengths and separation are scaled accordingly, provided $z_{R s} \ll\left|d_{s}-f\right|$ :

$$
\begin{gathered}
z_{R i} \simeq z_{R s} M^{2}, \\
z_{i} \simeq-z_{u} M^{2}, \\
w_{i} \simeq w_{s} M .
\end{gathered}
$$

After substitution in Eq. (C6) we see that the polarization properties on the image correspond to the demagnified properties of the source:

$$
\begin{array}{ll}
\psi(x, y, t, \Delta \omega) & =\phi\left(z_{u}\right)+k z_{u}-\frac{k r^{2}}{2 z_{i} M^{2}\left[1+\left(z_{R i} / z_{i}\right)^{2}\right]}+\frac{\Delta \omega t}{2} \\
\chi(x, y, t, \Delta \omega) & =0,
\end{array}
$$

where $r$ is the coordinate transverse to the optical axis on the image which is demagnified by a factor $M$.

[1] E. Saldin, E. Schneidmiller, and M. Yurkov, The Physics of Free Electron Lasers (Springer, Berlin Heidelberg, 2010).

[2] H. Rubinstein-Dunlop et al., Roadmap on Structured Light, J. Opt. 19, 013001 (2017).

[3] J. L. Ellis, K. M. Dorney, D. D. Hickstein, N. J. Brooks, C. Gentry, C. Hernández-García, D. Zusin, J. M. Shaw, Q. L. Nguyen, C. A. Mancuso et al., High harmonics with spatially varying ellipticity, Optica 5, 479 (2018).

[4] A. M. Beckley, T. G. Brown, and M. A. Alonso, Full Poincaré beams, Opt. Express 18, 10777 (2010).

[5] T. Bauer, P. Banzer, E. Karimi, S. Orlov, A. Rubano, L. Marrucci, E. Santamato, R. W. Boyd, and G. Leuchs, Observation of optical polarization Mobius strips, Science 347, 964 (2015).

[6] F. Weise, M. Pawłowska, G. Achazi, and A. Lindinger, Full control of polarization and temporal shape of ultrashort laser pulses transmitted through an optical fibre, J. Opt. 13, 075301 (2011).

[7] I. Zagorodnov, G. Feng, and T. Limberg, Corrugated structure insertion for extending the SASE bandwidth up to $3 \%$ at the European XFEL, Nucl. Instrum. Methods Phys. Res., Sect. A 837, 69 (2016).

[8] A. B. Temnykh, Delta undulator for Cornell energy recovery linac, Phys. Rev. Accel. Beams 11, 120702 (2008).

[9] P. Li, T. Wei, Y. Li, and J. Pflueger, Magnetic design of an Apple-X afterburner for the SASE3 undulator of the European XFEL, Nucl. Instrum. Methods Phys. Res., Sect. A 870, 103 (2017).

[10] K. J. Kim, A synchrotron radiation source with arbitrarily adjustable elliptical polarization, Nucl. Instrum. Methods Phys. Res., Sect. A 219, 425 (1984).

[11] K.-J. Kim, Circular polarization with crossed-planar undulators in high-gain FELs, Nucl. Instrum. Methods Phys. Res., Sect. A 445, 329 (2000).

[12] Y. K. Wu, N. A. Vinokurov, S. Mikhailov, J. Li, and V. Popov, High-Gain Lasing and Polarization Switch with a Distributed Optical-Klystron Free-Electron Laser, Phys. Rev. Lett. 96, 224801 (2006).

[13] H. Deng, T. Zhang, L. Feng, C. Feng, B. Liu, X. Wang, T. Lan, G. Wang, W. Zhang, X. Liu, J. Chen, M. Zhang, G. Lin, M. Zhang, D. Wang, and Z. Zhao, Polarization switching demonstration using crossed-planar undulators in a seeded free-electron laser, Phys. Rev. Accel. Beams 17, 020704 (2014).

[14] E. Ferrari, E. Allaria, J. Buck, G. De Ninno, B. Diviacco, D. Gauthier, L. Giannessi, L. Glaser, Z. Huang, M. Ilchen, G. Lambert, A. A. Lutman, B. Mahieu, G. Penco, C. Spezzani, and J. Viefhaus, Single shot polarization characterization of XUV FEL pulses from crossed polarized undulators, Sci. Rep. 5, 13531 (2015).

[15] J. Yan, J. M. Mueller, M. W. Ahmed, H. Hao, S. Huang, J. Li, V. N. Litvinenko, P. Liu, S. F. Mikhailov, V. G. Popov, M. H. Sikora, N. A. Vinokurov, and Y. K. Wu, Precision control of gamma-ray polarization using a crossed helical 
undulator free-electron laser, Nat. Photonics 13, 629 (2019).

[16] Y. Li, B. Faatz, and J. Pflueger, 3D Polarization properties for crossed-planar undulators, TESLA-FEL Report, 2010.

[17] P. Emma, K. Bane, M. Cornacchia, Z. Huang, H. Schlarb, G. Stupakov, and D. Walz, Femtosecond and Subfemtosecond X-Ray Pulses from a Self-Amplified SpontaneousEmission-Based Free-Electron Laser, Phys. Rev. Lett. 92, 074801 (2004).

[18] A. Marinelli, R. Coffee, S. Vetter, P. Hering, G. N. West, S. Gilevich, A. A. Lutman, S. Li, T. Maxwell, J. Galayda, A. Fry, and Z. Huang, Optical Shaping of X-Ray FreeElectron Lasers, Phys. Rev. Lett. 116, 254801 (2016).

[19] A. A. Lutman et al., Polarization control in an x-ray freeelectron laser, Nat. Photonics 10, 468 (2016).

[20] A. A. Lutman, T. J. Maxwell, J. P. MacArthur, M. W. Guetg, N. Berrah, R. N. Coffee, Y. Ding, Z. Huang, A. Marinelli, S. Moeller, and J. C. U. Zemella, Fresh-slice multicolour X-ray free-electron lasers, Nat. Photonics 10, 745 (2016).

[21] J. Feldbaus, E. L. Saldin, J. R. Schneider, E. A. Schneidmiller, and M. V. Yurkov, Possible application of $\mathrm{X}$-ray optical elements for reducing the spectral bandwidth of an X-ray SASE FEL, Nucl. Instrum. Methods Phys. Res., Sect. A 393, 162 (1997).

[22] S. Serkez, Design and optimization of the Grating Monochromator for Soft X-Ray Self-Seeding FELs, Ph.D. thesis, Universitaet Hamburg, 2015.

[23] D. Ratner et al., Experimental Demonstration of a Soft X-Ray Self-Seeded Free-Electron Laser, Phys. Rev. Lett. 114, 054801 (2015).
[24] E. A. Schneidmiller and M. V. Yurkov, Obtaining high degree of circular polarization at X-ray free electron lasers via a reverse undulator taper, Phys. Rev. Accel. Beams 16, 110702 (2013).

[25] S. Serkez, G. Geloni, V. Kocharyan, and E. Saldin, Circular polarization opportunities at the SASE3 undulator line of the European XFEL, arXiv:1608.08175.

[26] S. Reiche, GENESIS 1.3: A fully 3D time-dependent FEL simulation code, Nucl. Instrum. Methods Phys. Res., Sect. A 429, 243 (1999).

[27] Ocelot collaboration, Ocelot collaboration webpage: https://github.com/ocelot-collab/ocelot (2016).

[28] E. Schneidmiller and M. Yurkov, Reverse undulator tapering for polarization control at SASE3, European XFEL group meeting, http://edmsdirect.desy.de/ edmsdirect/file.jsp?edmsid=6754511 (2016).

[29] E. A. Schneidmiller and M. V. Yurkov, Reverse undulator tapering for polarization control at SASE3, European XFEL group meeting, Phys. Rev. Accel. Beams 16, 110702 (2013).

[30] S. Serkez, G. Geloni, and E. Saldin, Interference-based ultrafast polarization control at free electron lasers, in Proceedings of the 38th International Free Electron Laser Conference, FEL2017, Santa Fe, NM, USA (JACoW, Geneva, Switzerland, 2017), pp. 325-328.

[31] A. Scherz (private communication).

[32] M. Meyer (private communication).

[33] M. Born and E. Wolf, Principles of Optics Electromagnetic Theory of Propagation INterference and Diffraction of Light, 7th ed. (Cambridge University Press, Cambridge, England, 1999) p. 619. 